\title{
Explorando a influência integrada do Estímulo Docente sobre a intenção de uso das bibliotecas virtuais por estudantes de cursos à distância e presenciais no Brasil
}

Thaísa Bechelli Yamanaka*

Alexandre Cappellozza*

Artículo recibido:

25 de septiembre de 2015

Artículo aceptado:

28 de octubre de 2016

\section{Resumen}

Este estudio analizó los factores antecedentes que pueden influenciar la intención de uso de la biblioteca virtual de una institución de enseñanza superior. Se utilizó el Modelo de Aceptación de Tecnología-TAM (Davis, 1986) extendido por el Estímulo Docente y Hábito a fin de ampliar el conocimiento sobre las intenciones de uso de los usuarios de estos recursos educativos. Los datos fueron recolectados a través de 406 cuestionarios con estudiantes brasileños de cursos de enseñanza superior. Se concluye que el Estímulo Docente influye positivamente en la Facilidad de Uso, Utilidad Percibida y el Hábito de los alumnos de graduación sobre la intención de uso de la biblioteca virtual. Así, se constató que la influencia del profesor

* Universidad Metodista de São Paulo, Brasil. thaisa.yamanaka@metodista.br alexandre.cappellozza@metodista.br

INVESTIGACIÓN BIBLIOTECOLÓGICA, vol. 32, núm. 75, abril/junio, 2018, México, ISSN: 2448-8321. pp. 19-45 
es determinante sobre el uso de la biblioteca virtual por los estudiantes, lo que resalta la importancia de la orientación y recomendación de los interesados en el uso de esta herramienta tecnológica. La Utilidad Percibida es el factor más influyente en la intención de uso de esta tecnología. Además, el Hábito influye en la adopción de la biblioteca virtual de forma paralela a las percepciones sobre los beneficios que pueden obtenerse con el uso de esta tecnología. Los resultados muestran que los alumnos matriculados en cursos de enseñanza a distancia son más propensos al uso de estos recursos educativos comparados con los estudiantes de los cursos presenciales.

Palabras clave: Biblioteca Virtual; Aceptación de Tecnología; Enseñanza Superior

Exploring the comprehensive influence of Teaching Stimulus on the intention to use virtual libraries among students enrolled in classroom and distance learning courses in Brazil

Thaísa Bechelli-Yamanaka and Alexandre Cappellozza

\section{Abstract}

This study examines antecedent factors that may influence the intention to use the virtual library of college students enrolled in classroom and distance-learning courses. The study focuses on the Technology Acceptance Model - TAM (Davis, 1986) and the construct called Teaching Stimulus in order to expand knowledge of the impact of teaching on students' use of technological educational tools. Data were collected using 406 questionnaires answered by Brazilian undergraduate students. Findings demonstrate that Teaching Stimulus positively impacts ease of use, perceived usefulness and habits of students regarding their intention to use the Virtual Library. As such, this research emphasizes the importance of guidance and recommendations for expanding the use of technological educational resources by students. Moreover, the study concludes that perceived usefulness is the most important factor influencing the intention to use these technologies. Results show that students enrolled in distance-learning courses are more inclined to use these educational resources than students enrolled in classroom courses.

Keywords: Virtual Library; Technology Acceptance; Undergraduate Education 


\section{INTRODUÇÃO}

Com o avanço da tecnologia em todas as áreas de conhecimento, principalmente a partir dos anos 90, diferentes perspectivas para o gerenciamento de recursos de informação passaram a ser discutidas. Nesta época, houve a ampliação do acesso à internet para grande parte da população, o que possibilitou o aumento da comunicação entre os indivíduos, alterando as formas de busca e utilização da informação (Gomes, 2000).

Esta ampliação do acesso à internet possibilitou também a ascensão ao ensino superior para muitos indivíduos que, por algum motivo, não puderam cursar uma faculdade. Foi assim que no século XX a Educação a Distância (EAD) com o apoio dos avanços das novas Tecnologias da Informação e da Comunicação (TDIC), permitiu a inclusão de muitas pessoas no ensino superior (Preti, 1996).Para atender esta grande demanda de alunos no ensino superior, um dos serviços educacionais que as instituições disponibilizam ao seu corpo discente são as tradicionais bibliotecas.

Com a mudança no formato de organizar e disponibilizar informações, as bibliotecas atingiram diferentes perspectivas para o gerenciamento de recursos de informação. Uma destas perspectivas é que as bibliotecas puderam alcançar seu formato virtual. A biblioteca virtual apresenta-se como uma possível quebra no paradigma de tratamento e disseminação de informações representadas pelas atividades, recursos e serviços da "biblioteca tradicional" (Marchiori, 1997).

A biblioteca virtual trata-se de uma plataforma na web onde os usuários acessam livros no formato digital. Ela pode ser caracterizada como um instrumento que integra a infraestrutura básica da ciência que apoia o desenvolvimento científico, possibilitando que o usuário online possa ter ao seu alcance os recursos necessários que contribuam nos seus estudos como livros, revistas eletrônicas, etc. (Gomes, 2004).

A biblioteca virtual pode ser um importante instrumento de apoio ao ensino que as instituições estão disponibilizando aos seus alunos tanto da modalidade EAD, quanto para seus alunos da modalidade presencial. Entretanto, apenas fornecer esta ferramenta de estudos não é suficiente para que seu corpo discente busque ampliar seus conhecimentos, mas também é importante que este aluno tenha a iniciativa, estímulo e incentivo do professor em adotar esta tecnologia da informação.

Para analisar os motivos que levam os alunos a adotem a biblioteca virtual, torna-se fundamental identificar e entender os fatores que levam os indivíduos a adotarem uma determinada tecnologia da informação. Para isso, diversos estudos foram desenvolvidos a fim de identificar os fatores relacionados na 
decisão e intenção dos indivíduos quanto à aceitação e uso de tecnologias de informação (Venkatesh, Thong e Xu, 2012; Venkatesh et al., 2003; Cappellozza, 2014; Löbler, Visentini e Vieira, 2006).

Com base nesse contexto, este artigo identificará e analisará os fatores antecedentes que podem influenciar a intenção de uso da biblioteca virtual dos alunos de graduação da educação a distância (EAD) e dos alunos do ensino presencial de uma Instituição de Ensino Superior (IES) que dispõe de uma biblioteca virtual.

\section{ReFERENCIAL TEÓRICO}

Desde o século passado a tecnologia faz parte da vida da maioria das pessoas, proporcionando maior interação entre elas em tempo real, sem a necessidade de deslocamento físico. Através desta interação e com o avanço das Tecnologias Digitais de Informação e Comunicação (TDIC), foi possível a disseminação de informações, ou seja, expandir o conhecimento da população através dos recursos tecnológicos, como por exemplo, a internet. Por isso torna-se importante que as pessoas estejam familiarizadas com as TDIC, pois elas potencializam o aprendizado dos indivíduos e a sua permanente atualização (Amem e Nunes, 2006).

Devido ao maior acesso da internet pela população, foi possível integrar as TDIC nos processos educacionais, uma vez que elas estão presentes e influentes na vida social de muitas pessoas (Almeida, 2003).

A tecnologia também é uma realidade no cotidiano de alunos e professores. Com isso, a educação tecnológica é fato para as novas gerações e, como as instituições de ensino são parte integrante da cultura da sociedade, elas devem estar atentas para a leitura das linguagens tecnológicas, pois a universidade enquanto instituição social responsável pela formação de alunos, necessita estar ligada no contexto das práticas contemporâneas que atendam as exigências da educação do século XXI (Axt, 2000). Uma dessas exigências que surgiu no século passado e continua em crescente expansão é a Educação a distância (EAD).

A EAD se distingue da modalidade de ensino presencial por ser uma forma de ensino que utiliza a comunicação tecnológica, substituindo a interação pessoal que ocorre em sala de aula pelo conjunto de recursos didáticos com o apoio de uma instituição e tutoria que proporcionam uma aprendizagem independente e flexível (Aretio, 1994).

Com o passar dos anos, a educação a distância tem sido considerada um dos temas de grande relevância para a academia brasileira, pois de acordo 
com Censo da Educação Superior de 2006, em 2003 eram 49 mil estudantes nessa modalidade e em 2006 passaram a ser 207 mil, ou seja, houve um crescimento em $315 \%$ em todo o país (Instituto Nacional, 2007).

De acordo com dados do Censo da Associação Brasileira de Ensino a Distância (ABED), em 2008 o número de alunos nessa modalidade aumentou para 760 mil, superando a casa de um milhão em 2009. Em 2012 esse número ultrapassou para mais de 5,7 milhões de alunos matriculados em diversos cursos da modalidade a distância (Associação Brasileira, 2013).

Analisando também os resultados do Censo da Educação Superior (2012) (Mercadante, 2012) houve um crescimento no número de matrículas nos cursos de graduação da modalidade EAD atingindo $14,6 \%$ do total do número de matrículas, portanto, houve uma maior adesão por parte da população que tem ido ao encontro desta modalidade de ensino.

Com esta crescente demanda de interessados nesta modalidade de ensino, os serviços educacionais também devem suprir as necessidades informacionais de seus usuários remotos. Como um dos serviços educacionais, temse a biblioteca, que também precisou se adaptar para atender seus usuários (Ribeiro, 2012).

Com o avanço das Tecnologias Digitais de Informação e Comunicação (TDIC), as bibliotecas adquiriram novas possibilidades de interação e disseminação do conhecimento, alcançando assim, a sua virtualidade (Lazzarin et al., 2010).

A biblioteca virtual trata-se de uma plataforma na web onde os usuários acessam livros no formato digital. Ela pode ser caracterizada como um instrumento que integra a infraestrutura básica da ciência que apoia o desenvolvimento científico, possibilitando que o usuário online possa ter ao seu alcance os recursos necessários que contribuam nos seus estudos como livros, revistas eletrônicas, etc. (Gomes, 2004).

Para que possa existir a biblioteca virtual é imprescindível a utilização da tecnologia da realidade virtual. Em outras palavras ela necessita que um software seja acoplado a um computador que reproduz o ambiente de uma biblioteca em duas ou três dimensões, criando um ambiente onde o usuário poderá circular entre as salas, selecionar qualquer livro que esteja disponível nesta biblioteca e, assim, poderá abri-lo e ler o conteúdo (Marchiori, 1997).

Outro conceito pode ser visto em Gomes (2004) que se refere à biblioteca virtual como um serviço on-line de informação especializada, que foi criado para atender as novas exigências da pesquisa acadêmica, principalmente quanto à sua agilidade para obter a informação e comunicação aos seus usuários. A biblioteca virtual é utilizada tanto por alunos da EAD quanto por alunos do ensino presencial. 
Uma das características que diferenciam estes dois grupos de estudantes é que para os alunos do ensino presencial os professores que estão sempre presentes fisicamente, em horário limitado e determinado pelas instituições (Lobo Neto, 1999). Além disso, em função destes alunos disporem de maiores encontros presenciais, tendem a apresentarem um perfil menos pró-ativo que o aluno EAD na busca pelo conhecimento. Um dos fatores que levam estes alunos a serem pouco estimulados a tomarem tal iniciativa, pode ser justificado devido aos professores presenciais estarem habituados em apenas transmitir o conhecimento e não a incentivá-los à sua busca. Já o aluno da educação a distância, visto que não dispõe de um professor presencial e também por ser um aluno habituado quanto ao uso intensivo da tecnologia, tende a buscar ferramentas que lhe permita acesso ao conteúdo de forma digital (Vidal, 2002).

Uma das formas dos alunos buscarem a expansão de seus conhecimentos no formato digital está na biblioteca virtual que, quando bem estruturada, pode oferecer recursos, produtos e serviços remotamente com eficácia e qualidade. Buscar este recurso é interessante para que o aluno não necessite recorrer e nem se deslocar constantemente à biblioteca do polo de apoio presencial (Pereira e Sanches, 2010).

Contudo, existem outras variáveis que envolvem o processo de aprendizagem, desta forma, é necessário entender os elementos que os compõe para que o aluno possa ter uma aprendizagem efetiva, principalmente na relação entre as pessoas envolvidas, ou seja, na própria relação entre professor-aluno, uma vez que esta influência é considerada fundamental no processo de ensino-aprendizagem (Stacciarini e Esperidião, 1999).

Para que ocorra o processo de ensino-aprendizagem, Moreira (1986) menciona que existem quatro elementos dentro deste processo: professor, aluno, conteúdo e as variáveis ambientais, onde esta última pode ser considerada o ambiente o qual o aluno está inserido (instituição de ensino). Para Santos (2001) o ponto principal do processo de melhoria da qualidade de ensino nas instituições de nível superior, deve ser o entendimento e interação desses quatro elementos do ensino-aprendizagem. Teixeira (2002) argumenta que o aluno é responsável pela construção do seu conhecimento, ou seja, ele deve ter autonomia mostrando-se corresponsável no decorrer de seu percurso acadêmico. Para Freire (1996: 25) "quem ensina aprende ao ensinar e quem aprende ensina ao aprender”. Assim, o aluno aprende quando há uma estreita relação entre ensinar e aprender.

Seguindo esta linha de pensamento pode-se notar a importância do papel dos professores na construção desse processo de ensino-aprendizagem, pois eles são parte integrante do processo educativo e na formação das gerações 
e padrões de sociedade (Libâneo, 1994). Nesta sociedade contemporânea, muitos alunos ainda acreditam que seu papel no processo de aprendizagem é de apenas ouvir os professores, memorizar conceitos e desejar respostas, ou seja, muitos ainda demonstram atitude passiva em sala de aula (Santos, 2001). Para reverter este processo, é necessário que o docente incentive e estimule o aluno para que ele tenha a motivação e autonomia necessária na busca dos conhecimentos, pois o professor repassa informações onde cada aluno deverá aproveitar este momento para interpretar dados e informações, transformando-os em conhecimentos. O papel do professor é mantê-los curiosos e motivados para que sejam interessados na busca de conhecimentos, uma vez que ninguém transfere conhecimento, mas sim, transferem dados e informações (Poletti, 2002).

Portanto, entende-se que o processo educativo é interativo e se concretiza através das relações entre e professor e aluno, onde a figura do professor torna-se extremamente relevante devido à mediação que o professor faz entre conhecimento e alunos, proporcionando assim, a apropriação ativa do conhecimento (De Vasconcelos et al., 2005). Assim, visto que o professor tem um papel fundamental na vida dos seus alunos, motivando-os e incentivando-os na busca pelo conhecimento, torna-se relevante um estudo sobre o quanto o professor em seu papel de educador, estimula seus alunos na busca pelo conhecimento. Uma das formas deste incentivo é fazer com o que seus alunos utilizem as tecnologias disponíveis como a biblioteca virtual, mas para isso, faz-se necessário entender os motivos que levam os indivíduos a adorarem, ou não, uma determinada tecnologia (Davis, 1989).

Os estudos de adoção de tecnologia possibilitaram o desenvolvimento de teorias que auxiliam no entendimento e compreensão dos fatores que determinam a intenção e o comportamento de uso de determinados sistemas (Venkatesh, Thong y Xu, 2012). Provindas da área da Psicologia, a Teoria Racional do Trabalho - TRA (Fishbein e Ajzen, 1975) e da Teoria do Comportamento Planejado - TPB (Ajzen, 1991) foram utilizadas como base para a criação de diversos modelos de adoção de tecnologia, dentre os diversos modelos existem, tem-se o Modelo de Aceitação de Tecnologia - TAM proposto por Davis (1986). Este modelo é considerado um dos mais influentes e utilizados pelos pesquisadores para descrever a aceitação dos indivíduos por uma determinada tecnologia, estudando a influência de fatores humanos em sua adoção (Dillon e Morris, 1996; Venkatesh et al., 2003). Além disso, este modelo possui uma forte base teórica e apoio empírico através de validações, aplicações e replicações do seu modelo, sendo testado com diferentes amostras e situações, mostrando-se assim, ser válido e confiável (Costa Filho, Pires e Hernandez, 2007). 
O TAM foi desenvolvido com a intenção de explicar o que determina uma pessoa a aceitar ou rejeitar um sistema de informação, buscando entender através da utilidade e da facilidade de uso percebida, o comportamento do usuário (Davis, Bagozzi e Warshaw, 1989). Ilustra-se o modelo TAM na Figura 1.

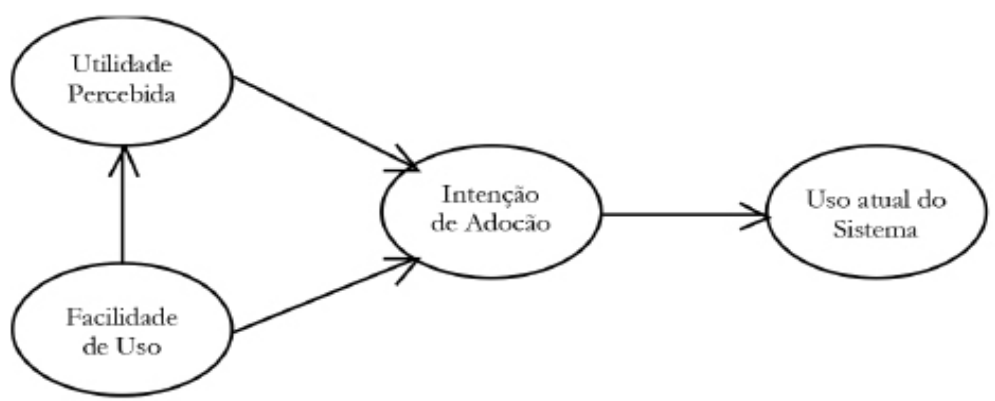

Figura 1. Modelo de Aceitação de Tecnología - TAM (Davis, Bagozzi e Warshaw, 1989)

A Utilidade Percebida se refere ao grau no qual uma pessoa acredita que a utilização de um determinado sistema pode contribuir para a melhoria de seu desempenho no trabalho (Davis, 1986). A melhoria do desempenho poderia ser atingida, por exemplo, quando se leva menos tempo para cumprir uma tarefa ou para produzir produtos de maior qualidade. A Facilidade de Uso se refere ao grau no qual uma pessoa acredita que utilizar um determinado sistema estaria livre de esforço (Davis, 1986). Neste estudo o construto Facilidade de Uso busca analisar o grau de percepção do aluno com a biblioteca virtual em relação à sua facilidade, ou seja, verificará se este aluno acredita que pode ser fácil utilizar a biblioteca virtual, contribuindo assim, com seus estudos.

Utilizando os construtos Utilidade Percebida e Facilidade de Uso para verificar a relação como fatores antecedentes da intenção de uso da biblioteca virtual, elaboram-se as seguintes hipóteses:

H1: A Utilidade Percebida influencia positivamente a Intenção de Uso da Biblioteca Virtual.

H2: A Facilidade de Uso influencia positivamente a Intenção de Uso da Biblioteca Virtual.

Os dois construtos (Utilidade Percebida e Facilidade de Uso) procuram verificar se os indivíduos tendem a usar ou não uma tecnologia com o objetivo de melhorar seu desempenho no trabalho (Utilidade Percebida), porém mesmo 
que essa pessoa entenda que uma determinada tecnologia é útil, sua utilização poderá ser prejudicada se o uso for muito complicado, de modo que o esforço não compense o uso (Davis, 1986). Com base nisso, busca-se verificar se os alunos que percebem que é fácil utilizar a biblioteca virtual, também perceberão sua utilidade. Desta forma, cria-se a terceira hipótese desta pesquisa.

H3: A Facilidade de Uso percebida influencia positivamente sua Utilidade Percebida.

O TAM é um modelo válido e robusto que tem sido vastamente utilizado que permite uma aplicabilidade mais ampla, ou seja, neste modelo é possível incluir outras variáveis externas que permitam ampliar os estudos sobre a adoção de tecnologia individual (King e He, 2006). Neste estudo foram incluídas as variáveis bábito e estímulo docente.

Sob a perspectiva da Tecnologia da Informação, o hábito tem por objetivo analisar a influência das ações automáticas tomadas por indivíduos no que se refere ao uso da tecnologia (Venkatesh, Thong e Xu, 2012). Desta forma, elabora-se a seguinte hipótese:

H4: O Hábito influencia positivamente a Intenção de Uso da Biblioteca Virtual.

O Estímulo Docente se refere ao apoio, incentivo e orientação do professor aos seus alunos, uma vez que cabe ao professor ser o mediador entre conhecimento e aluno (De Vasconcelos et al., 2005). A fim de verificar a influência do Estímulo Docente na intenção de uso da Biblioteca Virtual, este construto será avaliado como antecedente dos construtos Utilidade Percebida, Facilidade de Uso e Hábito. Para isso, apresentam-se as seguintes hipóteses.

H5: O Estímulo Docente influencia positivamente a Utilidade Percebida da Biblioteca Virtual.

H6: O Estímulo Docente influencia positivamente a Facilidade de Uso da Biblioteca Virtual.

H7: O Estímulo Docente influencia positivamente o Hábito de uso da Biblioteca Virtual.

Os alunos da EAD possuem maior contato com a tecnologia, devido à utilização de um ambiente virtual de aprendizagem nos seus estudos (Ribeiro, 
Mendonça e Mendonça, 2007). Com isso, pressupõe-se que estes alunos possam apresentar diferenças de percepção na utilidade percebida, na facilidade de uso e também no hábito. Portanto, com a finalidade de explorar também a modalidade de ensino, ou seja, se há diferença na intenção de uso da biblioteca virtual entre os estudantes de graduação do ensino presencial e da EAD, foi incluída ao modelo conceitual desta pesquisa a variável "modalidade de ensino" para moderar a relação das variáveis "facilidade de uso", "utilidade percebida" e "hábito" com a "intenção de uso" da biblioteca virtual.

As variáveis moderadoras são fatores que impactam na relação entre as variáveis dependentes e independentes (Venkatesh et al., 2003). Deste modo, para moderar esta relação, foram criadas as seguintes hipóteses:

H8a: A modalidade de ensino modera a relação entre o Hábito e a Intenção de Uso da Biblioteca Virtual.

H8b: A modalidade de ensino modera a relação entre a Facilidade de Uso e a Intenção de Uso da Biblioteca Virtual.

H8c: A modalidade de ensino modera a relação entre Utilidade Percebida e a Intenção de Uso da Biblioteca Virtual.

Elaboradas as hipóteses de pesquisa a serem testadas empiricamente e, pressupondo o relacionamento entre os construtos nos fatores de influência da intenção de uso da biblioteca virtual, apres enta-se o modelo conceitual de pesquisa conforme Figura 2.

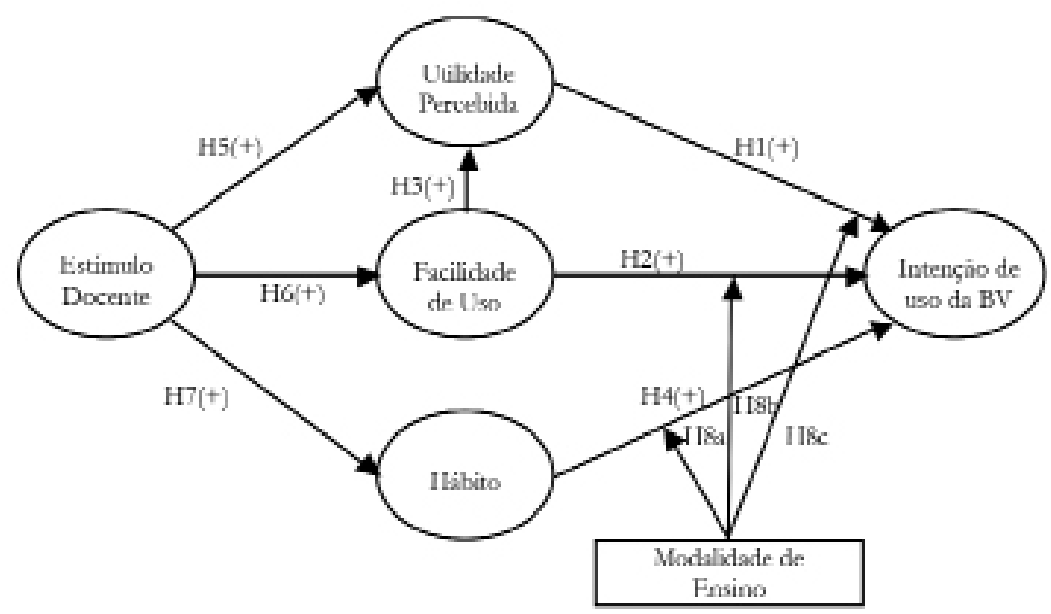




\section{Procedimentos metodológicos}

Esta pesquisa de abordagem quantitativa é de corte transversal único de caráter confirmatório. A coleta de dados empíricos foi realizada por meio de surveys onde as informações foram coletadas por meio de um questionário que utilizou a escala Likert de sete pontos.

Para aplicação do instrumento de medida, foram validados 406 questionários sendo 132 questionários respondidos por alunos do ensino a distância e 274 questionários respondidos por alunos do ensino presencial. A amostra mínima calculada no software G*Power 3.1. 9 foi de 68 casos, mas a recomendação de Ringle, Silva e Bido (2014) é que, para que o modelo seja consistente, é interessante utilizar o dobro ou o triplo desse valor. Desta forma, o tamanho da amostra final (406) superou a recomendação mínima necessária.

Analisando os dados obtidos em relação ao gênero, a amostra de 406 participantes onde 202 respondentes são do gênero feminino e 204 do gênero masculino. A idade média foi de 26 anos com desvio padrão de 7,92. A idade do menor respondente é de 17 anos enquanto que a maior idade é de 57 anos. Essa variação aponta que a amostra é composta de jovens e adultos de meia idade, ou seja, há um encontro de diferentes gerações sinalizando que a idade pode não ser impeditivo para cursar o ensino superior.

Em relação aos cursos dos respondentes, em sua maioria, 64,7\% concentram-se nos cursos de Gestão de Recursos Humanos, Administração e Logística. Nos cursos tecnólogos de Gestão de Recursos e Humanos e Logística houve uma quantidade maior de respondentes devido ao instrumento de pesquisa ter sido aplicado a vários períodos na modalidade do ensino presencial e a distância. Já no curso de Administração apesar do instrumento ter sido aplicado apenas na modalidade EAD, este curso por ser bacharelado é composto por 8 períodos, enquanto que, nos cursos tecnólogos, os períodos variam entre 4 a 5 .

Do total dos respondentes $92,4 \%$ já fizeram uso da biblioteca virtual, sendo que o maior porcentual que já utilizaram decorre dos alunos da EAD com $97 \%$ contra $90,1 \%$ dos alunos do ensino presencial, o que também mostra que a amostra está habilitada a participar da pesquisa sobre a biblioteca virtual pelo contato prévio com a tecnologia estudada.

A Tabela 1 reúne as médias dos itens de cada construto e apresenta as médias gerais das frequências das respostas por construto com suas respectivas médias e desvios padrão. 
Tabela 1. Frequência média de respostas por construto

\begin{tabular}{|l|c|c|}
\hline \multicolumn{1}{|c|}{ Construto } & Médida & Desvio Padrão \\
\hline Facilidade de Uso & 4,69 & 1,38 \\
\hline Utilidade Percebida & 4,84 & 1,26 \\
\hline Estímulo Docente & 4,22 & 1,51 \\
\hline Hábito & 3,95 & 1,52 \\
\hline Intenção de Uso & 5,01 & 1,29 \\
\hline
\end{tabular}

Analisando as médias gerais dos construtos, destaca-se com a média mais alta a Intenção de Uso com média 5,01 e desvio padrão de 1,29. Em relação a menor média, aparece o Hábito com 3,95 de média e desvio padrão de 1,52. Para verificar a normalidade de distribuição dos dados, foi aplicado o teste de diagnóstico de normalidade Kolmogorov-Smirnov.

Tabela 2. Teste de normalidade

\begin{tabular}{|l|c|l|}
\hline Construtos & p-valor & Resultado \\
\hline Facilidade de Uso & 0,027 & Distribuição não é normal \\
\hline Utilidade Percebida & 0,004 & Distribuição não é normal \\
\hline Estímulo Docente & 0,003 & Distribuição não é normal \\
\hline Hábito & 0,074 & Distribuição normal \\
\hline Intenção de Uso & 0,003 & Distribuição não é normal \\
\hline
\end{tabular}

Após os resultados dos testes de normalidade, contata-se que a distribuição dos dados para os construtos Facilidade de Uso, Utilidade Percebida, Estímulo Docente e Intenção de Uso, não pode ser considerada como normal. Já o construto Hábito apresentou distribuição normal, visto que o p-valor foi maior que 0,05 .

Dados os resultados obtidos dos testes de normalidade e, para se verificar se existe diferença significante entre as medianas em relação às percepções da biblioteca virtual pela modalidade de ensino, foi utilizado o teste de Mann-Whitney.

A Tabela 3, apresentam-se as medianas dos escores médios dos indicadores de cada construto, desvio padrão e a significância do teste de Mann-Whitney para cada construto por modalidade de ensino. 
Tabela 3. Teste de comparação entre grupos (EAD e Presencial)

\begin{tabular}{|c|c|c|c|c|c|c|}
\hline Variável & Modalidade & $\mathrm{N}$ & Mediana & $\begin{array}{l}\text { Desvio } \\
\text { padrão }\end{array}$ & Teste W & $\mathrm{p}$-valor \\
\hline \multirow[t]{2}{*}{ Facilidade de Uso } & EAD & 132 & 5,50 & 1,74 & \multirow[t]{2}{*}{31976,0} & \multirow[t]{2}{*}{0,0000} \\
\hline & Presencial & 274 & 4,50 & 1,13 & & \\
\hline \multirow{2}{*}{$\begin{array}{l}\text { Utilidade } \\
\text { Percebida }\end{array}$} & EAD & 132 & 5,60 & 1,45 & \multirow[t]{2}{*}{33319,5} & \multirow[t]{2}{*}{0,0000} \\
\hline & Presencial & 274 & 4,60 & 1,10 & & \\
\hline \multirow[t]{2}{*}{ Estímulo Docente } & EAD & 132 & 5,33 & 1,47 & \multirow[t]{2}{*}{34246,0} & \multirow[t]{2}{*}{0,0000} \\
\hline & Presencial & 274 & 3,83 & 1,41 & & \\
\hline \multirow[t]{2}{*}{ Hábito } & EAD & 132 & 4,75 & 1,66 & \multirow[t]{2}{*}{32848,0} & \multirow[t]{2}{*}{0,0000} \\
\hline & Presencial & 274 & 4,00 & 1,36 & & \\
\hline \multirow[t]{2}{*}{ Intenção de Uso } & EAD & 132 & 6,00 & 1,32 & \multirow[t]{2}{*}{34208,0} & \multirow[t]{2}{*}{0,0000} \\
\hline & Presencial & 274 & 5,00 & 1,19 & & \\
\hline
\end{tabular}

Como a significância dos resultados dos testes de Mann-Whitney foram menores que 0,05 , pode-se afirmar que há diferenças significativas nos dois grupos de estudantes, pressupondo que as percepções sobre a biblioteca virtual dos alunos da EAD são diferentes em relação às percepções dos alunos do ensino presencial.

Os alunos da EAD apresentaram maior percepção da Facilidade de Uso em relação aos alunos do ensino presencial. Este resultado pode estar relacionado ao fato deste grupo de alunos possuírem maior contato com a tecnologia, uma vez que em seus estudos utiliza-se um ambiente virtual de aprendizagem (Ribeiro, Mendonça e Mendonça, 2007).

A Utilidade Percebida também se mostrou mais presente no grupo dos alunos EAD, assim percebe-se que os alunos da modalidade EAD reconhecem que obterão mais benefícios sobre o uso da biblioteca virtual.

Percebe-se que Estímulo Docente consolida-se também no grupo EAD, sugerindo assim, que os professores que atuam na educação a distância estimulam com maior intensidade seus alunos na adoção da biblioteca virtual.

Pressupondo que os alunos da EAD estão mais habituados com o uso da tecnologia para utilização nos seus estudos, uma vez que eles utilizam uma plataforma tecnológica em seus estudos de forma rotineira, o Hábito de uso da biblioteca virtual apresentou-se com maior veemência neste mesmo grupo de alunos. 
Por fim, a Intenção de uso também se mostrou mais acentuado no grupo dos alunos da EAD em relação aos alunos do ensino presencial. Sugerindo assim, que a maior possibilidade de adoção da biblioteca virtual encontra-se nos alunos da educação a distância.

Para examinar a validade convergente e discriminante dos construtos, foi utilizada a Análise Fatorial Confirmatória no modelo estrutural (Hair et al., 2005). A validade discriminante consiste no grau em que dois conceitos similares são distintos e a validade convergente avalia o grau em que duas medidas do mesmo conceito estão correlacionadas (Hair et al., 2005; Hair et al., 2013).

A Tabela 4 apresenta o resultado da Análise Fatorial Confirmatória, onde todos os construtos apresentam indicadores com cargas altas em suas variáveis latentes e cargas baixas nas demais variáveis latentes, indicando boa validade discriminante e convergente (Chin, 2010).

Tabela 4. Cargas fatoriais nos construtos

\begin{tabular}{|c|c|c|c|c|c|c|}
\hline Construto & Variável & ED & FAC & HAB & UTI & IU \\
\hline Estímulo Docente & ED_1 & 0,830 & 0,399 & 0,458 & 0,486 & 0,459 \\
\cline { 2 - 7 } & ED_2 & 0,826 & 0,438 & 0,450 & 0,457 & 0,374 \\
\cline { 2 - 7 } & ED_3 & 0,895 & 0,408 & 0,538 & 0,509 & 0,474 \\
\cline { 2 - 7 } & ED_4 & 0,877 & 0,508 & 0,484 & 0,537 & 0,492 \\
\cline { 2 - 7 } & ED_5 & 0,912 & 0,469 & 0,505 & 0,530 & 0,502 \\
\cline { 2 - 7 } & ED_6 & 0,795 & 0,449 & 0,556 & 0,470 & 0,449 \\
\hline Facilidade de Uso & FAC_1 & 0,367 & 0,751 & 0,444 & 0,421 & 0,365 \\
\cline { 2 - 7 } & FAC_2 & 0,458 & 0,872 & 0,676 & 0,666 & 0,606 \\
\cline { 2 - 7 } & FAC_3 & 0,428 & 0,891 & 0,579 & 0,579 & 0,475 \\
\cline { 2 - 7 } & FAC_4 & 0,507 & 0,886 & 0,680 & 0,633 & 0,558 \\
\hline Hábit0 & HAB_1 & 0,486 & 0,620 & 0,819 & 0,604 & 0,536 \\
\cline { 2 - 7 } & HAB_2 & 0,488 & 0,546 & 0,876 & 0,645 & 0,632 \\
\cline { 2 - 7 } & HAB_3 & 0,496 & 0,660 & 0,857 & 0,625 & 0,594 \\
\cline { 2 - 7 } & HAB_4 & 0,506 & 0,582 & 0,837 & 0,646 & 0,668 \\
\hline & UTI_1 & 0,370 & 0,556 & 0,565 & 0,790 & 0,578 \\
\cline { 2 - 7 } & UTI_2 & 0,557 & 0,579 & 0,649 & 0,890 & 0,729 \\
\cline { 2 - 7 } & UTI_3 & 0,567 & 0,578 & 0,691 & 0,900 & 0,757 \\
\cline { 2 - 7 } & UTI_4 & 0,497 & 0,653 & 0,655 & 0,866 & 0,699 \\
\hline Utilidade Percebida & & & & & \\
\hline
\end{tabular}




\begin{tabular}{|l|c|c|c|c|c|c|}
\hline Intenção de Uso & IU_1 & 0,414 & 0,526 & 0,601 & 0,728 & 0,863 \\
\cline { 2 - 7 } & $\mathrm{IU} \_2$ & 0,411 & 0,484 & 0,632 & 0,713 & 0,874 \\
\cline { 2 - 7 } & $\mathrm{IU} 33$ & 0,502 & 0,527 & 0,568 & 0,686 & 0,855 \\
\cline { 2 - 7 } & $\mathrm{IU} 4$ & 0,444 & 0,417 & 0,560 & 0,472 & 0,658 \\
\hline
\end{tabular}

Outro indicador utilizado para validade convergente é a Variância Média Extraída (AVE). De acordo com Fornell e Larcker (1981) e Hair, Ringle e Sarstedt (2011), o critério para validação do indicador é apresentar um valor superior a 0,5 . Todos os indicadores ficaram acima dos valores satisfatórios de acordo com a Tabela 5 .

Tabela 5. Variância Extraída, Confiabilidade e Consistência dos construtos

\begin{tabular}{|l|c|c|c|}
\hline \multicolumn{1}{|c|}{ Construtos } & $\begin{array}{c}\text { VariânciaMédia } \\
\text { Extraída (AVE) }\end{array}$ & $\begin{array}{c}\text { Confiabilidade } \\
\text { Composta }\end{array}$ & $\begin{array}{c}\text { Consistência Interna } \\
\text { (Alpha de Cronbach) }\end{array}$ \\
\hline Estímulo Docente & 0,735 & 0,943 & 0,927 \\
\hline Facilidade de Uso & 0,726 & 0,914 & 0,874 \\
\hline Hábito & 0,718 & 0,911 & 0,869 \\
\hline Utilidade Percebida & 0,744 & 0,921 & 0,885 \\
\hline Intenção de Uso & 0,668 & 0,888 & 0,830 \\
\hline
\end{tabular}

Para avaliar o modelo de mensuração, outro indicador utilizado é a confiabilidade composta (Hair et al., 2013). Este indicador descreve o grau em que os indicadores representam o construto latente em comum. $\mathrm{O}$ valor de referência aceitável é 0,70 .

Para a validade convergente é utilizada a consistência interna sendo que um alto valor de consistência interna no construto indica que todas as variáveis representam o mesmo construto latente.

O Alpha de Cronbach avalia a consistência interna, e seu valor varia de 0 a 1 , sendo que altos valores indicam alto nível de consistência. Para estudos exploratórios, valores entre 0,60 e 0,70 são considerados aceitáveis; já para estudos em estágios mais avançados, valores entre 0,70 e 0,90 são considerados satisfatórios (Fornell e Larcker, 1981; Nunally e Bernstein, 1994; Hair et al., 2013).

$\mathrm{Na}$ Tabela 5, mostra-se que todos os valores da confiabilidade composta estão dentro do estabelecido pelos autores.

A matriz de correlação estimada e a raiz quadrada da variância média extraída dos construtos (AVE) são utilizadas para verificar a validade discriminante entre construtos. Para Fornell e Larcker (1981), a raiz quadrada da AVE dos construtos deve ser maior que a correlação entre as variáveis 
latentes. A Tabela 6 apresenta os valores que são considerados adequados para validade discriminante. Em negrito, na diagonal, são apresentados os valores da raiz quadrada da AVE dos construtos.

Tabela 6. Matriz de correlação e raiz quadrada de AVE

\begin{tabular}{|l|l|l|l|l|c|}
\hline \multicolumn{1}{|c|}{ Construtos } & \multicolumn{1}{c|}{ ED } & \multicolumn{1}{c|}{ FAC } & HAB & UTI & IU \\
\hline Estímulo Docente & $\mathbf{0 , 8 5 7}$ & & & & \\
\hline Facilidade de Uso & 0,521 & $\mathbf{0 , 8 5 2}$ & & & \\
\hline Hábito & 0,583 & 0,709 & $\mathbf{0 , 8 4 7}$ & & \\
\hline Utilidade Percebida & 0,583 & 0,686 & 0,744 & $\mathbf{0 , 8 6 3}$ & \\
\hline Intenção de Uso & 0,536 & 0,599 & 0,720 & 0,805 & $\mathbf{0 , 8 1 7}$ \\
\hline
\end{tabular}

Para análise da significância dos indicadores foi utilizada a técnica bootstrapping (Efron e Tibshirani, 1998). A reamostragem utilizada no estudo foi de 5.000 amostras com reposição de 406 casos, de acordo com as recomendações de Hair et al. (2013).

Tabela 7. Cargas fatoriais do modelo de medidas e estatística t de Student

\begin{tabular}{|l|c|c|c|c|}
\hline \multicolumn{1}{|c|}{ Variável } & Cargas (média) & Desvio Padrão & Desvio Padrão & $\begin{array}{c}\text { p-valor (bicau- } \\
\text { dal) }\end{array}$ \\
\hline ED_1<-ED & 0,181 & 0,009 & 21,194 & 0,000 \\
\hline ED_2<-ED & 0,181 & 0,009 & 19,263 & 0,000 \\
\hline ED_3<-ED & 0,197 & 0,006 & 31,563 & 0,000 \\
\hline ED_4<- ED & 0,205 & 0,008 & 25,469 & 0,000 \\
\hline ED_5<-ED & 0,203 & 0,007 & 30,725 & 0,000 \\
\hline ED_6<- ED & 0,199 & 0,009 & 21,003 & 0,000 \\
\hline FAC_1<-FAC & 0,224 & 0,015 & 14,614 & 0,000 \\
\hline FAC_2<- FAC & 0,326 & 0,014 & 23,955 & 0,000 \\
\hline FAC_3<-FAC & 0,290 & 0,010 & 29,796 & 0,000 \\
\hline FAC_4<-FAC & 0,327 & 0,014 & 24,051 & 0,000 \\
\hline HAB_1<-HAB & 0,272 & 0,010 & 26,260 & 0,000 \\
\hline HAB_2<-HAB & 0,301 & 0,009 & 32,309 & 0,000 \\
\hline HAB_3<-HAB & 0,291 & 0,008 & 35,143 & 0,000 \\
\hline HAB_4<-HAB & 0,316 & 0,010 & 30,448 & 0,000 \\
\hline IU_1<-IU & 0,331 & 0,011 & 30,900 & 0,000 \\
\hline
\end{tabular}




\begin{tabular}{|l|l|l|l|l|}
\hline IU_2<- IU & 0,331 & 0,011 & 29,575 & 0,000 \\
\hline IU_3<- IU & 0,313 & 0,011 & 28,494 & 0,000 \\
\hline IU_4<- IU & 0,240 & 0,016 & 15,385 & 0,000 \\
\hline UTI_1<- UTI & 0,247 & 0,009 & 27,131 & 0,000 \\
\hline UTI_2<- UTI & 0,301 & 0,008 & 38,381 & 0,000 \\
\hline UTI_3<- UTI & 0,308 & 0,008 & 40,327 & 0,000 \\
\hline UTI_4<- UTI & 0,300 & 0,008 & 37,929 & 0,000 \\
\hline
\end{tabular}

$\mathrm{Na}$ Tabela 8 apresentam-se os valores dos coeficientes entre os construtos e as estatísticas t de Student, estimados pela técnica de bootstrapping. Todos os valores dos relacionamentos apresentaram valo res de $t$ de Student superiores a 1,96 (nível de significância $=5 \%$ ).

Tabela 8. Coeficientes do modelo estrutural - entre construtos

\begin{tabular}{|l|c|c|c|c|}
\hline $\begin{array}{c}\text { Relação entre } \\
\text { Construtos }\end{array}$ & $\begin{array}{c}\text { Cargas } \\
\text { (média) }\end{array}$ & $\begin{array}{c}\text { Desvio } \\
\text { Padrão }\end{array}$ & Teste - T & $\begin{array}{c}\text { p-valor } \\
\text { (bicaudal) }\end{array}$ \\
\hline ED -> FAC & 0,522 & 0,039 & 13,436 & 0,000 \\
\hline ED -> HAB & 0,584 & 0,035 & 16,661 & 0,000 \\
\hline ED -> UTI & 0,311 & 0,048 & 6,450 & 0,000 \\
\hline FAC -> UTI & 0,523 & 0,053 & 9,907 & 0,000 \\
\hline HAB -> IU & 0,270 & 0,044 & 6,160 & 0,000 \\
\hline UTI -> IU & 0,604 & 0,048 & 12,560 & 0,000 \\
\hline
\end{tabular}

O coeficiente de determinação $\left(\mathrm{r}^{2}\right)$ indica quanto da variação total é comum aos elementos que constituem os pares analisados. Para Cohen (1988), valores de $\mathrm{r}^{2}$ iguais a $10 \%$ são considerados baixos, $30 \%$ são considerados médios e $50 \%$ são considerados altos. De acordo com os resultados apresentados na Tabela 9, nota-se que os valores de $\mathrm{r}^{2}$ dos construtos Hábito e Utilidade Percebida são considerados como médios e o valor da Intenção de Uso obteve um alto valor no poder de explicação, o que pode classificar como satisfatória a seleção dos construtos nesta pesquisa.

A técnica blindfolding foi utilizada para verificar a relevância preditiva do modelo. Os resultados de valor $\mathrm{Q}^{2}$ maiores que 0 indicam que os construtos exógenos têm relevância preditiva sobre os construtos endógenos em consideração. Todos os valores foram considerados acima de zero conforme apresenta a Tabela 9 . 
Tabela 9. Valores de $\mathrm{r}^{2} \mathrm{e} \mathrm{Q}^{2}$ dos construtos endógenos

\begin{tabular}{|l|c|c|c|}
\hline Construtos & $\mathbf{r}^{2}$ & $\begin{array}{c}\mathbf{r}^{2} \\
\text { ajustado }\end{array}$ & $\mathbf{Q}^{2}$ \\
\hline Facilidade de Uso & 0,271 & 0,270 & 0,450 \\
\hline Hábito & 0,340 & 0,338 & 0,243 \\
\hline Utilidade Percebida & 0,540 & 0,537 & 0,398 \\
\hline Intenção de Uso & 0,680 & 0,679 & 0,450 \\
\hline
\end{tabular}

O tamanho do efeito $\mathrm{f}^{2}$ permite verificar a contribuição de um construto exógeno sobre uma variável latente endógena. Os valores $\mathrm{f}^{2}$ de 0,02, 0,15 e 0,35 indicam, respectivamente, um efeito pequeno, médio e grande de um construto exógeno sobre um construto endógeno (Cohen, 1988). A Tabela 10 apresenta os valores de $\mathrm{f}^{2}$ e mostra que a maioria dos construtos apresenta um efeito entre médio e grande.

Tabela 10. Valores de $\mathrm{f}^{2}$

\begin{tabular}{|l|c|c|c|c|}
\hline \multicolumn{1}{|c|}{ Construtos } & FAC & HAB & UTI & IU \\
\hline Estímulo Docente & 0,373 & 0,515 & 0,152 & \\
\hline Facilidade de Uso & & & 0,435 & \\
\hline Hábito & & & & 0,103 \\
\hline Utilidade Percebida & & & & 0,507 \\
\hline
\end{tabular}

As hipóteses referentes à variável moderadora "modalidade de ensino" foram operacionalizadas como uma variável dummy, sendo o valor 0 correspondendo aos alunos da EAD e o valor 1 correspondendo ao grupo dos alunos do ensino presencial. Ambas foram testadas e rodadas em um novo modelo.

A Tabela 11 apresenta os valores dos coeficientes do moderador modalidade de ensino entre os construtos e as estatísticas $t$ de Student, estimados pela técnica de bootstrapping.

Tabela 11. Coeficientes do modelo estrutural entre construtos

\begin{tabular}{|l|c|c|c|c|}
\hline \multicolumn{1}{|c|}{ Moderador } & Cargas (média) & Desvio Padrão & Teste - T & p-valor (bicaudal) \\
\hline $\begin{array}{l}\text { Modalidade-> FAC } \\
->\text { IU }\end{array}$ & 0,064 & 0,053 & 1,246 & 0,213 \\
\hline $\begin{array}{l}\text { Modalidade-> HAB } \\
->\text { IU }\end{array}$ & 0,030 & 0,056 & 0,622 & 0,534 \\
\hline $\begin{array}{l}\text { Modalidade -> UTI } \\
->\text { IU }\end{array}$ & $-0,025$ & 0,055 & 0,519 & 0,604 \\
\hline
\end{tabular}


Todos os valores da moderação apresentaram valores de $t$ de Student inferiores a 1,96 (nível de significância = 0,05). Dessa forma, o moderador modalidade de ensino não obteve valores significantes, não confirmando as hipóteses associadas com esta variável.

A Figura 3 apresenta o modelo resultante da pesquisa com os valores das cargas fatoriais, coeficientes entre os construtos com suas respectivas significâncias e os valores de poder de explicação de cada construto.

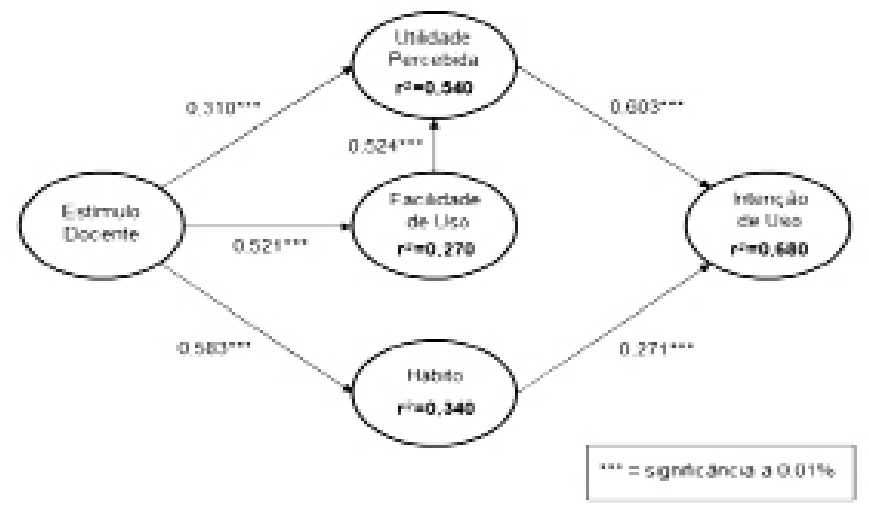

Figura 3. Síntese de resultados do modelo estrutural

Inicialmente nota-se que a estrutura do modelo TAM proposto por Davis (1986) foi validada parcialmente para esta aplicação, embora a confirmação de significância de resultados da estrutura de construtos do modelo TAM já tenha sido validada anteriormente por outros estudos de adoção de TI (Davis, Bagozzi e Warshaw, 1989; Adams, Nelson e Todd, 1992; Taylor e Todd, 1995; Szajna, 1996; Tarhini et al., 2015; Teo, 2010; Lin, Shih e Sher, 2007; Park, 2009; Cappellozza, 2013).

Neste estudo a Facilidade de Uso não teve efeitos significantes na Intenção de Uso. Com isso, nesta aplicação, a estrutura do modelo TAM original proposto por Davis (1986) não foi validada. Este resultado pode estar associado à experiência que o usuário possui com o sistema, ou seja, quanto mais tempo de uso com o sistema, pode-se atenuar a relação entre facilidade de uso e intenção comportamental, uma vez que este usuário já utiliza e conhece a ferramenta e pode não associar a facilidade de uso como um fator motivador ao uso (Venkatesh e Bala, 2008; Venkatesh et al., 2003).

Além disso, este resultado também pode estar associado pela falta de alternativas de uso da plataforma analisada, o que pode mascarar as relações entre construtos (Adams, Nelson e Todd, 1992). Uma outra possível 
justificativa dos resultados é a mediação da relação entre facilidade e intenção de uso pela utilidade percebida.

A Utilidade Percebida foi a variável que apresentou maior influência para a Intenção de Uso da Biblioteca Virtual. Este fato converge com os resultados obtidos por Davis (1986), Davis, Bagozzi e Warshaw (1989), Venkatesh et al. (2003) entre outros, comprovando-se o efeito direto da utilidade percebida na intenção uso e reafirmando que este construto possui a maior relevância na avaliação da aceitação de tecnologias de informação, conforme estudos desenvolvidos por Davis (1986).

Observa-se na Tabela 9 que a inclusão das variáveis externas Estímulo Docente e Hábito também resultou em um poder de explicação significativo na intenção de uso da biblioteca virtual. O resultado positivo e significante do Estímulo Docente com os construtos Utilidade Percebida, Facilidade de Uso e Hábito reafirma a importância do professor no papel de mediador entre alunos e a adoção tecnologias de informação e comunicação educacionais.

O resultado positivo e significante do efeito da variável Hábito demonstra favorecimento da sua utilização contínua e, como uma possível consequência, pode promover a intenção de uso e a respectiva adoção desta ferramenta de estudo.

Os coeficientes obtidos na análise do relacionamento entre a variável moderadora "modalidade de ensino" não foi significante, portanto não foram suportadas as hipóteses 8a, 8b e 8c. Deste modo o impacto do Hábito, da Facilidade de Uso e da Utilidade Percebida sobre a Intenção de Uso não foi moderada pela modalidade de ensino dos alunos de graduação.

Contudo, a qualidade do modelo conceitual desta pesquisa foi evidenciada pela avaliação dos $\mathrm{R}^{2}$ das variáveis latentes. A adição das variáveis externas ao modelo TAM proporcionou o poder de explicação de 68\% na intenção de uso da biblioteca virtual. Para Cohen (1988) resultados acima de $50 \%$ são considerados altos, portanto, nesta pesquisa os resultados em relação à intenção de uso da biblioteca virtual foram obtidos com êxito.

$\mathrm{Na}$ Tabela 12 é possível visualizar de forma sintética os resultados dos poderes de explicação de cada construto utilizado nesta pesquisa.

Tabela 12. Valores de $\mathrm{R}^{2}$ ajustados dos construtos

\begin{tabular}{|l|c|}
\hline Construtos & $\mathbf{R}^{2}$ ajustado \\
\hline Utilidade Percebida & 0,54 \\
\hline Intenção de Uso & 0,27 \\
\hline Hábito & 0,34 \\
\hline Intenção de Uso & 0,68 \\
\hline
\end{tabular}


A Tabela 13 apresenta s síntesis dos testes de hipóteses do estudo.

Tabela 13. Resultado dos testes de hipóteses

\begin{tabular}{|l|l|l|}
\hline Hipótese & & Resultado \\
\hline H1 & $\begin{array}{l}\text { A Utilidade Percebida influencia positivamente a } \\
\text { Intenção de Uso da Biblioteca Virtual }\end{array}$ & Confirmada \\
\hline H2 & $\begin{array}{l}\text { A Facilidade de Uso influencia positivamente a } \\
\text { Intenção de Uso da Biblioteca Virtual }\end{array}$ & Não confirmada \\
\hline H3 & $\begin{array}{l}\text { A Facilidade de Uso influencia positivamente sua } \\
\text { Utilidade Percebida }\end{array}$ & Confirmada \\
\hline H4 & $\begin{array}{l}\text { O Hábito influencia positivamente a Intenção de Uso } \\
\text { da Biblioteca Virtual }\end{array}$ & Confirmada \\
\hline H5 & $\begin{array}{l}\text { O Estímulo Docente influencia positivamente a } \\
\text { Utilidade Percebida da Biblioteca Virtual }\end{array}$ & Confirmada \\
\hline H6 & $\begin{array}{l}\text { O Estímulo Docente influencia positivamente a } \\
\text { Facilidade de Uso da Biblioteca Virtual }\end{array}$ & Confirmada \\
\hline H7 & $\begin{array}{l}\text { 0 Estímulo Docente influencia positivamente 0 } \\
\text { Hábito da Uso da Biblioteca Virtual }\end{array}$ & Confirmada \\
\hline H8a & $\begin{array}{l}\text { A modalidade de ensino modera a relação entre } \\
\text { Hábito e Intenção de Uso da Biblioteca Virtual }\end{array}$ & Não Confirmada \\
\hline H8b & $\begin{array}{l}\text { A modalidade de ensino modera a relação entre } \\
\text { Facilidade de Uso e Intenção de Uso da Biblioteca } \\
\text { Virtual }\end{array}$ & Não confirmada \\
\hline H8c & $\begin{array}{l}\text { A modalidade de ensino modera a relação entre } \\
\text { Utilidade Percebida e Intenção de Uso da Biblioteca } \\
\text { Virtual }\end{array}$ & Não confirmada \\
\hline
\end{tabular}

\section{Conclusões}

Para esta pesquisa foram selecionadas variáveis do modelo TAM (Davis, 1986) com o objetivo de propiciar a consideração de elementos associados à intenção de uso da Biblioteca Virtual que é utilizada por estudantes de graduação de uma Instituição de Ensino, a fim de aprimorar o conhecimento no processo de adoção individual desta ferramenta. Neste estudo também foram incluídas características individuais e influência do professor por serem consideradas relevantes no estudo desta tecnologia em questão.

O construto Hábito foi inserido no modelo TAM a fim de verificar se o hábito exerce influência na intenção de uso da biblioteca virtual, uma vez que o hábito é definido como uma repetição de um comportamento e pode influenciar as intenções individuais de uso de TI (Lankton, Wilson e Mao, 2010). 
O Estímulo Docente foi inserido no modelo conceitual desta pesquisa como fator antecedente da Facilidade de Uso, da Utilidade Percebida e do Hábito e se mostrou como um fator preponderante à adoção da biblioteca virtual pelos alunos. Por meio da aplicação de 406 questionários individuais, foi possível validar os relacionamentos entre os construtos componentes do modelo de pesquisa desenvolvido para o estudo da intenção de uso da biblioteca virtual.

Com a adição dos construtos Hábito e Estímulo Docente no modelo TAM, foi possível aprimorar o conhecimento do processo de adoção da biblioteca virtual, obtendo assim, evidências sobre as percepções individuais dos usuários desta tecnologia.

Nesta pesquisa evidenciou-se que o hábito influencia positivamente a intenção de uso da biblioteca virtual. Este resultado confirma com resultados de outros estudos que consideraram a influência do hábito à adoção de tecnologias de informação (Limayem, Hirt e Cheung, 2007; Guinea e Markus, 2009; Venkatesh, Thong e Xu, 2012; Cappellozza, 2013; Dias, Zwicker e Vincentin, 2003).

De acordo com os resultados verificou-se que o Estímulo Docente se apresentou como o fator que mais influencia o Hábito de uso da biblioteca virtual pelos alunos. Assim, se o professor incentivar, orientar e indicar seus alunos a utilizarem a biblioteca virtual, naturalmente estes alunos desenvolverão o hábito de utilizá-la.

Além disso, os resultados mostraram que o Estímulo Docente também apresenta influência positiva e significante na Utilidade Percebida e na Facilidade de Uso. Assim, a figura do professor torna-se relevante para que o aluno perceba a utilidade e a facilidade da ferramenta. Portanto, o estímulo docente torna-se um importante um fator que antecede outros fatores na intenção de uso da biblioteca virtual.

Corroborando com o Modelo de Aceitação de Tecnologia - TAM proposto por Davis (1986) o construto Utilidade Percebida influencia positivamente a intenção de uso do sistema, assim como a Facilidade de Uso influencia a Utilidade Percebida da biblioteca virtual, ou seja, se o aluno perceber que é fácil utilizar a biblioteca virtual, também perceberá sua utilidade.

Entende-se como limitação desta pesquisa, o local de obtenção da amostra do estudo, pois a coleta de dados se restringiu apenas a uma instituição e uma plataforma de biblioteca virtual. Portanto, para estudos futuros, sugere-se abranger outras instituições de ensino e diferentes plataformas tecnológicas.

A ausência de significância do relacionamento entre a facilidade e intenção e uso da biblioteca virtual é um resultado que exige cautela na sua generalização a outros estudos e merece investigação futura, pois o resultado 
difere de estudos anteriores que analisam este modelo de adoção de tecnologia. Assim, uma das possibilidades da obtenção deste resultado pode ser a mediação plena da utilidade na relação entre a facilidade de uso percebida e a intenção de uso da tecnologia. Outra possibilidade de explicação destes resultados é a falta de alternativas de uso da plataforma analisada, o que pode mascarar as relações entre construtos (Adams, Nelson e Todd, 1992).

Por fim, sugere-se também uma análise por região dos alunos da EAD, a fim de verificar se há diferenças nas percepções e intenções de uso da biblioteca virtual que podem ser ocasionadas por diferenças regionais.

\section{REFERÊNCIAS}

Adams, D. A., R. R. Nelson e P. A. Todd. 1992. "Perceived usefulness, ease of use, and usage of information technology: A replication". MIS Quarterly [S.I.] 16 (2): 227-247.

Ajzen, I. 1991. "The theory of planned behavior". Organizational behavior and buman decision processes 50 (2): 179-211.

Almeida, M. E. B. 2003. "Educação a distância na internet: abordagens e contribuições dos ambientes digitais de aprendizagem”. Educação e Pesquisa 29 (2): 327 340. Acesso em: 09 set. 2014. http://www.scielo.br/pdf/ep/v29n2/a10v29n2.pdf

Amem, B. M. V. e L. C. Nunes. 2006. “Tecnologias de informação e comunicação: contribuições para o processo interdisciplinar no ensino superior”. Revista Brasileira de Educação Médica 30 (3): 171-180.

Aretio, L. G. 1994. Educación a distancia boy. Universidad Nacional de Educación a Distancia: UNED.

Associação Brasileira de Educação a Distância. 2013. CensoEaD. BR 2013/2014. Acesso em 22 jan. 2015. http://www.abed.org.br/site/pt/midiateca/censo_ ead/1272/2014/10/censoead.br_2013/2014

Axt, M. 2000. "Tecnologia na Educação, Tecnologia para Educação: um texto em construção”. Informática na educação: teoria E prática 3 (1): 51-62.

Cappellozza, A. 2013. Modelo estendido de adoção da tecnologia de comunicação pessoal de voz pela internet. Tese de Doutorado, Fundação Getulio Vargas.

Cappellozza, A. 2014. "Grátis não é o suficiente: estudo da adoção individual da telefonia pela Internet”, em XXXVIII Encontro da Associação Nacional de Pós-Graduação e Pesquisa em Administração (EnANPAD), Rio de Janeiro.

Chin, W. W. 2010. "How to write Up and report PLS analyses", em Handbook of partial least squares: Concepts, methods and applications, V. E. Vinzi et al. (ed.), 655690. Berlin: Springer.

Cohen, J. 1988. Statistical power analysis for the behavioral sciences, 2. ed. Hillsdale, NJ: Erlbaum.

Costa Filho, B. A., P. J. Pires e J. M. C. Hernandez. 2007. "Modelo Technology Acceptance Model - TAM aplicado aos Automated Teller Machines - ATM's”. RAI 4 (1): 40-56. 
Davis, F. D. 1986. A technology acceptance model for empirically testing new end-user information systems: Theory and results. Tese de Doutorado, Massachusetts Institute of Technology.

Davis, F. D. 1989. "Perceived usefulness, perceived ease of use, and user acceptance of information technology”. MIS Quarterly (Minneapolis, MN) 13 (3): 319-339.

Davis, F. D., R. P. Bagozzi e P. R. Warshaw. 1989. "User acceptance of computer technology: A comparison of two theoretical models". Management Science 35 (8): 982-1003.

De Vasconcelos, A. A., A. C. G. da Silva, J. de Souza Martins e L. J. Soares. 2005. “A presença do diálogo na relação professor-aluno”. Trabalho apresentado em V Colóquio Internacional Paulo Freire. Recife, Brasil.

Dias, M. C., R. Zwicker e I. C. Vincentin. 2003. "Análise do Modelo de Aceitação de Tecnologia de Davis”. Spei (Curitiba) 4 (2): 15-23.

Dillon, A. e M. Morris. 1996. "User acceptance of new information technology: theories and models". ARIST 31:3-32.

Efron, B. e R. J. Tibshirani. 1998. An introduction to the bootstrap. EUA: CRC Press.

Fishbein, M. e L. Ajzen. 1975. Belief, attitude, intention, and behavior: An introduction to theory and research. Reading, Mass.: Addison-Wesley.

Fornell, C. e D. F. Larcker. 1981. "Evaluating structural equation models with unobservable variables and measurement error". Journal of Marketing Research 18: 39-50.

Freire, P. 1996. Pedagogia da autonomia: saberes necessários à prática educativa, 13. ed. Rio de Janeiro: Paz e Terra.

Gomes, S. L. R. 2000. Bibliotecas Virtuais: informação e comunicação para a pesquisa cientifica. Tese de Doutorado, IBICT-UFRJ-ECO.

Gomes, S. L. R. 2004. "Biblioteca virtual: um novo território para a pesquisa científica no Brasil”. Data Gama Zero: revista de Ciência da Informação 5 (6): 1-13. Acesso em: 16 out. 2014. http://www.dgz.org.br/dez04/Art_05.htm

Guinea, A. O. e M. L. Markus. 2009. "Why break the habit of a lifetime? Rethinking the roles of intention, habit, and emotion in continuing information technology use". MIS Quarterly 33 (3): 433-444.

Hair, J. F., R. E. Anderson, R. L. Tatham e W. C. Black. 2005. Análise Multivariada de Dados, 5. ed. Porto Alegre: Bookman.

Hair, J. F., G. T. M. Hult, C. M. Ringle e M. Sarstedt. 2013. A Primer on Partial Least Squares Structural Equation Modeling (PLS-SEM). Thousand Oaks: SAGE Publications.

Hair, J. F., C. M. Ringle e M. Sarstedt. 2011. "PLS-SEM: Indeed a silver bullet”. The Journal of Marketing Theory and Practice 19 (2): 139-152.

Instituto Nacional de Estudos e Pesquisas Educacionais Anisio Teixeira. 2007. Educação a Distância cresce mais ainda entre os cursos superiores. Acesso em: 10 out. 2016. http://portal.inep.gov.br/c/journal/view_article_content?groupId=10157\&articleId $=15449$ \&version $=1.0$

King, W. R. e J. He. 2006. "A meta-analysis of the technology acceptance model". Information E management 43 (6): 740-755.

Lankton, N. K., E. V. Wilson e E. Mao. 2010. "Antecedents and determinants of information technology habit”. Information E Management 47 (5): 300-307.

Lazzarin, F. A., E. B. D. Carvalho, E. D. Valério, N. L. D. Carmo e R. G. D. Santos. 2010. "Tecnologias de informação: o processo de utilização dos serviços 
das bibliotecas virtuais correlatos ao profissional bibliotecário”. Trabalho apresentado em XXXIII Encontro Nacional de Estudantes de Biblioteconomia, Documentação, Gestão e Ciência da Informação. Paraíba, Brasil.

Libâneo, J. C. 1994. Didática. São Paulo: Cortez.

Limayem, M., S. G. Hirt e C. M. K. Cheung. 2007. "How habit limits the predictive power of intention: The case of information systems continuance". MIS Quarterly 31 (4): 705-737.

Lin, C. H., H. -Y. Shih e P. J. Sher. 2007. "Integrating technology readiness into technology acceptance: The tram model”. Psychology E Marketing 24 (7): 641-657.

Löbler, M., M. S. Visentini e K. M. Vieira. 2006. "A aceitação do comércio eletrônico explicada pelos modelos TAM e combinados". Trabalho apresentado em Anais do XXX Encontro da Associação Nacional de Pós-Graduação e Pesquisa em Administração. Salvador, Brasil.

Lobo Neto, F. J. S. 1999. Educação a Distância: Regulamentação, Condições de Exxito e Perspectivas. Acesso em: 05 jul. 2014. http://www.feg.unesp.br/ saad/zip/RegulamentacaodaEducacaoaDistancia_lobo.htm

Marchiori, P. Z. 1997. “'Ciberteca' ou biblioteca virtual: uma perspectiva de gerenciamento de recursos de informação”. Ciência da Informação 26 (2). http://dx. doi.org/10.1590/S0100-19651997000200002

Mercadante, A. 2012. Censo da educação superior 2012. Disponível em Acesso em: 10 out. 2016. http://www.andifes.org.br/wp-content/files_flutter/1379600228mercadante.pdf

Moreira, D. A. 1986. "Elementos para um plano de melhoria do ensino universitário ao nível de instituição". Revista IMES III (9): 28-32.

Nunally, J. C. e I. Bernstein. 1994. Psychometric theory. New York: McGraw-Hill.

Park, S. Y. 2009. "An Analysis of the Technology Acceptance Model in Understanding University Students' Behavioral Intention to Use e-Learning”. Educational technology \& society 12 (3): 150-162.

Pereira, F. A. e A. L. A. R. Sanches. 2010. Bibliotecas digitais e virtuais no contexto da EAD: serviços online para usuários remotos. Acesso em: 28 set. 2014. http://www. sibi.ufrj.br/snbu2010/pdfs/orais//final_500.pdf

Poletti, A. 2002. "O professor e a gestão do conhecimento". Profissão mestre (São Paulo): 22-23.

Preti, O. 1996. "Educação a Distância: uma prática educativa mediadora e mediatizada". Educação a Distância: inícios e indícios de um percurso. NEAD/IE/UFMT. Cuiabá: UFMT.

Ribeiro, E. N., G. A. A. Mendonça, e A. F. Mendonça. 2007. "A importância dos ambientes virtuais de aprendizagem na busca de novos domínios na EAD”. Trabalho apresentado em: Anais do $13^{\circ}$ Congresso Internacional de Educação a Distância. Curitiba, Brasil.

Ribeiro, R. M. R. 2012. "A tecnologia da informação e comunicação (TIC): fator condicionante da inovação em bibliotecas universitárias”. Revista Digital Biblioteconomia e Ciência da Informação 9 (2): 4-8. Acesso em: 14 out. 2014. http://www. sbu.unicamp.br/seer/ojs/index.php/sbu_rci/index

Ringle, C. M., D. Silva e D. Bido. 2014. "Modelagem de Equações Estruturais com utilização do Smartpls”. Revista Brasileira de Marketing13 (2): 56-73. 
Santos, S. C. 2001. "O processo de ensino-aprendizagem e a relação professor-aluno: Aplicação dos 'sete princípios para a boa prática na educação de Ensino superior"”. Caderno de pesquisas em administração 8 (1): 69-82.

Stacciarini, J. M. R. e E. Esperidião. 1999. "Repensando estratégias de ensino no processo de aprendizagem”. Rev Latino-am Enfermagem 7 (5): 59-66.

Szajna, B. 1996. "Empirical evaluation of the revised technology acceptance model". Management Science 42 (1): 85-92.

Tarhini, A., M. Hassouna, M. S. Abbasi e J. Orozco. 2015. “Towards the Acceptance of RSS to Support Learning: An empirical study to validate the Technology Acceptance Model in Lebanon". Electronic Journal of e-Learning 13 (1): 30-41.

Taylor, S. e P. A. Todd. 1995. "Understanding information technology usage: A test of competing models". Information Systems Research 6 (2): 144-176.

Teixeira, M. 2002. Prática docente e autonomia do aluno: uma relação a ser construída em cursos de graduação. Tese de doutorado, Educação e Currículo, PUCSP, São Paulo.

Teo, T. 2010. "A path analysis of pre-service teachers' attitudes to computer use: Applying and extending the Technology Acceptance Model in an educational context". Interactive Learning Environments 18 (1): 65-79.

Venkatesh, V. e H. Bala. 2008. "Technology acceptance model 3 and a research agenda on interventions”. Decision sciences 39 (2): 273-315.

Venkatesh, V., M. G. Morris, G. B. Davis e F. D. Davis. 2003. "User Acceptance of Information Technology: Toward a Unified View”. MIS Quarterly 27 (3): 425-478.

Venkatesh, V., J. Y. L. Thong e X. Xu. 2012. "Consumer Acceptance and Use of Information Technology: Extending The Unified Theory Of Acceptance And Use Of Technology". MIS Quarterly 36 (1): 157-178.

Vidal, E. 2002. Ensino a distância VS Ensino Tradicional. Porto: Universidade Fernando Pessoa.

Para citar este texto:

Bechelli Yamanaka, Thaísa y Alexandre Cappellozza. 2018.

"Explorando a influência integrada do Estímulo Docente

sobre a intenção de uso das bibliotecas virtuais por estudantes de cursos à distância e presenciais no Brasil”. Investigación Bibliotecológica: archivonomía, bibliotecología e información 32 (75): 19-45.

http://dx.doi.org/10.22201/iibi.24488321xe.2018.75.57941 
EXPLORANDO A INFLUÊNCIA INTEGRADA DO ESTÍMULO DOCENTE SOBRE A INTENÇÃO...

\begin{tabular}{|c|}
\hline Apêndice \\
\hline Questões aplicadas aos respondentes \\
\hline $\begin{array}{l}\text { Facilidade de uso } \\
\text { É fácil localizar os livros que preciso na biblioteca virtual } \\
\text { É fácil utilizar a biblioteca virtual nos meus estudos } \\
\text { Eu acho fácil navegar na biblioteca virtual para fazer o que preciso } \\
\text { Minha interação com a biblioteca virtual é clara e compreensivel }\end{array}$ \\
\hline $\begin{array}{l}\text { Utilidade Percebida } \\
\text { Utilizar a biblioteca virtual me permite realizar tarefas de forma mais rápida } \\
\text { Utilizar a biblioteca virtual melhora meu desempenho nos estudos } \\
\text { Utilizar a biblioteca virtual aumenta minha eficácia nas tarefas do curso } \\
\text { A biblioteca virtual é útil em meus estudos e trabalhos acadêmicos } \\
\text { Utilizar a biblioteca virtual me deixa com mais tempo livre para outras atividades }\end{array}$ \\
\hline $\begin{array}{l}\text { Estímulo Docente } \\
\text { Os professores me incentivam a utilizar a biblioteca virtual } \\
\text { Os professores ensinam/orientam em como navegar na biblioteca virtual } \\
\text { Os professores solicitam a utilização da biblioteca virtual } \\
\text { Os professores indicam livros que estão disponíveis na biblioteca virtual } \\
\text { Os professores recomendam leituras dos livros disponíveis na biblioteca virtual } \\
\text { A resolução das tarefas solicitadas pelos professores depende dos livros da biblioteca virtual }\end{array}$ \\
\hline $\begin{array}{l}\text { Hábito } \\
\text { Eu utilizo a biblioteca virtual de forma automática } \\
\text { Utilizar a biblioteca virtual é um hábito para mim } \\
\text { Utilizar a biblioteca virtual é natural para mim } \\
\text { Eu não penso duas vezes para utilizar a biblioteca virtual quando necessito consultar um livro }\end{array}$ \\
\hline $\begin{array}{l}\text { Intenção de uso } \\
\text { Eu pretendo utilizar a biblioteca virtual nos meus estudos } \\
\text { Eu tenho a intenção de utilizar a biblioteca virtual sempre que possível } \\
\text { Eu prevejo utilizar a biblioteca virtual nos próximos semestres } \\
\text { Eu pretendo utilizar a biblioteca virtual ao invés da biblioteca tradicional }\end{array}$ \\
\hline
\end{tabular}

